[0212-7199 (2006) 23: 7; pp 310-316] ANALES DE MEDICINA INTERN Copyright (C) 2006 ARAN EDICIONES, S.L.

AN. MED. InTERNA (Madrid) Vol. 23, N. ${ }^{\circ}$, pp. 310-316, 2006

\section{Características clínicas, epidemiológicas y factores asociados a mortalidad en los pacientes hospitalizados por infecciones de piel y partes blandas. Resultados del estudio multicéntrico STIMG (Soft Tissue Infections Malacitan Group).}

\author{
F. SALGADO ORDÓÑEZ1 ${ }^{1}$ J. VILLAR JIMÉNEZ², A. HIDALGO CONDE ${ }^{3}$, \\ A. VILLALOBOS SÁNCHEZ ${ }^{2}$, J. DE LA TORRE LIMA ${ }^{4}$, J. AGUILAR GARCÍA ${ }^{4}$, \\ I. DA ROCHA COSTA ${ }^{1}$, M. Á. GARCÍA ORDÓÑEZ ${ }^{5}$, E. NUÑO ÁLVAREZ ${ }^{6}$, \\ C. RAMOS CANTES ${ }^{6}$, M. MARTÍN PÉREZ
}

Servicios de Medicina Interna. ${ }^{1}$ Hospital de la Serranía (Ronda). ${ }^{2}$ Hospital Carlos Haya. ${ }^{4}$ Hospital Costal del Sol (Marbella). ${ }^{3}$ Hospital Clínico Universitario Virgen de la Victoria. ${ }^{5}$ Hospital de Antequera (Antequera). ${ }^{6}$ Hospital de la Axarquia. Málaga

EPIDEMIOLOGICAL CHARACTERISTICS AND MORTALITY RISK FACTORS IN PATIENTS ADMITTED IN HOSPITALS WITH SOFT TISSUE INFECTIONS. A MULTICENTRIC STIMG (SOFT TISSUE INFECTIONS MALACITAN GROUP) STUDY RESULTS

\section{RESUMEN}

Objetivos: Describir las características de los pacientes hospitalizados con infecciones de piel y partes blandas (IPPB) y analizar las variables de los que fallecen durante el ingreso para definir grupos de riesgo.

Metodo: Análisis retrospectivo de las historias clínicas de todos los pacientes que fueron dados de alta con IPPB durante el año 2002, en los hospitales públicos de la provincia de Málaga. Se excluyen infecciones secundarias a pie diabético, cirugía, quemados, úlceras por decúbito y la celulitis orbitaria. Se analizaron variables clínicas, bioquímicas, indicación y validez diagnóstica de pruebas microbiológicas y de imagen, así como el tratamiento antibiótico empírico iniciado y su correlación con las recomendaciones terapéuticas establecidas.

Resultados: Analizamos 391 ingresos de 374 pacientes. La celulitis fue el diagnóstico más frecuente $(69,3 \%)$. Se realizaron pruebas de imagen en el $51,6 \%$ de los casos. El antibiótico más empleado fue la amoxicilina con ácido clavulánico (39\%). Fallecieron 27 (6,7\%), el 40,7\% por el proceso séptico en sí. Todos los fallecidos, tenían algún tipo de enfermedad crónica asociada. Las únicas variables analíticas que se asociaron de forma significativa a mayor mortalidad fueron las proteínas totales $(55 \pm 9 \mathrm{~g} / \mathrm{L}$ vs. $63 \pm 8 \mathrm{~g} / \mathrm{L} ; \mathrm{p}=0,0231)$ y los concentraciones séricas de albúmina $(22 \pm 7 \mathrm{~g} / \mathrm{L}$ vs. $29 \pm 7 \mathrm{~g} / \mathrm{L} ; \mathrm{p}=0,0125)$.

Conclusiones: Las celulitis es la IPPB que mayor número de ingresos genera en nuestro medio. Apreciamos una sobreutilización de pruebas de imagen y una desviación sobre las recomendaciones terapéuticas y el tratamiento aplicado en nuestros hospitales. La mortalidad global observada es baja y afecta de manera exclusiva a pacientes con mal estado nutricional al ingreso, enfermedades crónicas o infecciones profundas.

PALABRAS CLAVE: Infecciones de partes blandas. Mortalidad hospitalaria. Pronóstico.

\section{ABSTRACT}

Objectives: To describe the characteristics of patients admitted in hospitals with soft tissue infections, and analyse the variables whose died, in order to define risk groups.

Method: retrospective analysis of medical reports of all patient admitted during 2002 year for soft tissue infections in public malacitans hospitals. We excluded the patient with soft tissue infections associated with burns, surgery, pressure ulcers, and orbit cellulitis. We analysed clinical, biochemical variables and indications for yields and imaging tests, so the empiric antibiotic treatment established and its correlations with practice guidelines.

Results: We analysed 391 admissions of 374 patients. Cellulitis was the most frequent diagnosis (69.3\%). We did imaging tests in $51.6 \%$. In $94.3 \%$ of cases were treated with empirics antibiotics. The most prescribed drug was amoxiciline plus clavulanate (39\%). 27 patients died, $40.7 \%$ of them for septic cause. All deceased patients had chronic diseases. The only biochemical parameters associated with mortality were serum proteins and albumina $(55 \pm 9 \mathrm{~g} / \mathrm{L} v \mathrm{~s} .63 \pm 8 \mathrm{~g} / \mathrm{L} ; \mathrm{p}=0.0231)$ and (22 $\pm 7 \mathrm{~g} / \mathrm{L}$ vs. $29 \pm 7 \mathrm{~g} / \mathrm{L} ; \mathrm{p}=0.0125)$ respectively.

Conclusions: Cellullitis are the most frequent soft tissue infections that requires admissions in hospitals. We overuse imaging test and don't follow the practice guidelines recommendations in antibiotic therapy. Primary soft issue infection's mortality is low and it's restricted to people with chronic illness, deep infections and bad nutritional status.

KEY WORDS: Soft tissue infections. Hospital mortality. Outcome assessment.

Salgado Ordóñez F, Villar Jiménez J, Hidalgo Conde A, Villalobos Sánchez A, de la Torre Lima J, Aguilar García J, da Rocha Costa I, García Ordóñez M Á, Nuño Álvarez E, Ramos Cantes C, Martín Pérez M. Características clínicas, epidemiológicas y factores asociados a mortalidad en los pacientes hospitalizados por infecciones de piel y partes blandas. Resultados del estudio multicéntrico STIMG (Soft Tissue Infections Malacitan Group). An Med Interna (Madrid) 2006; 23: 310-316. 


\section{INTRODUCCIÓN}

Consideramos como infección de piel y partes blandas (IPPB) a todo proceso séptico que interesa a la epidermis, dermis y a los tejidos subyacentes, lo que incluye a la grasa subcutánea, fascias aponeuróticas y tejido muscular. De este modo clasificamos a las IPPB en virtud de estructura anatómica afectada, microorganismos causales y cuadro clínico, aunque a veces por la extensión de la IPPB es difícil de delimitar y un mismo microorganismo puede originar distintos cuadros, lo que hace que esta clasificación sea más académica que real (1). Por otra parte las IPPB constituyen un motivo de consulta frecuente en la practica clínica, aunque el impacto sanitario de estos procesos es desconocido, ya que existen pocos trabajos acerca de la incidencia y mortalidad de las IPPB $(2,3)$, y se centran sobre todo en infecciones necrosantes $(4,5)$ o en colectivos de riesgo (6). De hecho, y a tenor de los resultados de la búsqueda bibliográfica realizada (MEDLINE. 1964-2005), éste es el primer estudio sobre IPPB de estas características que se realiza en nuestro país.

Así pues, nos propusimos describir las características clínicas, epidemiológicas y mortalidad de los pacientes que fueron dados de alta de los hospitales públicos malagueños y presentaron infecciones primarias de partes blandas.

\section{MATERIAL Y MÉTODO}

Se trata de un estudio multicéntrico, retrospectivo de todos los pacientes que fueron dados de alta de forma consecutiva con IPPB durante el año 2002, en los hospitales del Sistema Sanitario Público Andaluz (SSPA) de la provincia de Málaga. Lo que incluye a 6 centros hospitalarios con un total de 2.674 camas y que atienden a una población de 1.287.017 habitantes (7).

Analizamos las historias clínicas de los pacientes con el diagnóstico de erisipela, ectima, celulitis, fascitis, gangrena, o miositis infecciosa siguiendo la clasificación CIE-9. Se excluyeron los pacientes con el diagnóstico infección de partes blandas secundarias a pie diabético, complicación postquirúrgica, quemados, ulceras por decúbito o por presión y la celulitis orbitaria, por considerar que estas entidades presentan características distintivas. Para homogenizar el contenido de la búsqueda los servicios de documentación clínica de los distintos centros participantes se coordinaron estableciendo los mismos criterios de codificación.

Se consideró que el paciente presentaba comorbilidad cuando constaba en los antecedentes personales al menos una de las siguientes patologías: Diabetes mellitus (DM), insuficiencia cardiaca (IC), enfermedad pulmonar obstructiva crónica (EPOC), hepatopatía crónica (HC), cáncer (neoplasia previa no controlada o no curada), insuficiencia renal crónica (IRC) (concentraciones séricas de creatinina mayor a $140 \mathrm{mmol} / \mathrm{L}$ ), obesidad (índice de masa corporal mayor a 35), o malnutrición. Se consideraron factores predisponentes la existencia de traumatismo previo, puerta de entrada (intertrigo, excoriación, etc), uso de drogas por vía parenteral (UDVP), insuficiencia venosa (varices, síndrome postfleblítico, linfedema en los antecedentes o en la exploración), arteriopatía periférica (claudicación intermitente, embolismos arteriales, intervención de by-pass o ausencia de pulsos en la exploración), uso de corticoides (dosis equivalente a $20 \mathrm{mg}$ prednisona/día durante más de una semana previa al ingreso), o estados de inmunosupresión (pacientes que habían recibido trasplante de órganos sólidos, inmunosupresores, eran alcohólicos, estaban malnutridos, infectados por el VIH con cifras de linfocitos CD4+ inferiores a $500 / \mathrm{mL}$ o tuvieran cualquier enfermedad asociada a inmunosupresión celular o humoral como discrasias sanguíneas, síndrome nefrótico, etc.). Se consideró al paciente como institucionalizado si provenía de una comunidad cerrada (cárcel, orfanato, hospital de crónicos, residencia de ancianos, etc.).

Se anotaron las constantes vitales al ingreso, existencia de necrosis cutánea a la exploración inicial, así como determinaciones analíticas básicas: leucocitos, proteínas totales, albuminemia y concentraciones séricas de creatinina.

Se registraron los resultados de los estudios microbiológicos practicados, hemocultivos y cultivos de la lesión, ya fueran por punción aspiración, biopsia o toma de exudado, excluyéndose del análisis los que fueron considerados como contaminados por el facultativo responsable del paciente en su momento. También se analizaron la indicación y resultados de las pruebas de imagen que se realizaron de la zona afecta por IPPB para estimar su valor predictivo positivo en el diagnóstico.

Se recogió el tipo de antibiótico empírico iniciado, la demora en el inicio del tratamiento, así como duración del mismo y se comparó el esquema escogido con las recomendaciones de la Sociedad Española de Enfermedades Infecciosas y Microbiología Clínica (8). También se procedió a analizar si el paciente requería intervención quirúrgica. Por último se registró el número de reingresos y si se producía el éxitus durante el siguiente año.

Los resultados recogidos se analizaron de forma univariada mediante el método de la Chi-cuadrado, para variables cualitativas, $t$ de Student y análisis de la varianza para cuantitativas. Se utilizó para su procesamiento el programa Epi Info $2000^{\mathrm{TM}}$ versión 1.1 .

\section{RESULTADOS}

Se analizaron 374 historias de pacientes que originaron 391 altas por IPPB. Esta cifra representa un 0,4\% de los ingresos y el $0,5 \%$ de las estancias de ese año en la provincia (9). Por diagnósticos, la celulitis fue la IPPB más frecuente con un $69,3 \%$, y las extremidades la región anatómica más afectada $(60,1 \%)$. Las infecciones odontogénicas motivaron el 22,5\% de los ingresos $(n=88)$, provenientes en su totalidad del Servicio de Cirugía Maxilofacial.

El rango de edad fue de 3 a 88 años. La gran mayoría de los pacientes ingresaban por la propia IPPB y adquirían la infección en la comunidad, 87 y $97 \%$ de los casos respectivamente. Casi la mitad de los pacientes $(48,9 \%)$ tenía alguna enfermedad crónica y el $76,1 \%$ factores predisponentes. Las características de los pacientes ingresados se resumen en la Tabla I.

Se realizaron técnicas de imagen en el 51,6\% de los ingresos $(n=202)$, con un total de 230 pruebas radiológicas practicadas. La rentabilidad diagnóstica de las mismas se recoge en la Tabla II. Se tomaron muestras para cultivar en 


\begin{tabular}{ll}
\multicolumn{2}{c}{ TABLA I } \\
\multicolumn{2}{c}{ CARACTERÍsTICAS DE LOS EPISODIOS INCLUIDOS EN EL } \\
\multicolumn{2}{c}{ ESTUDIO } \\
\hline Variable & $N(\%)$ \\
\hline Género hombre & $230(58,8)$ \\
Edad media (años) & 50,3 \\
Estancia media (días) & 8,4 \\
Motivo ingreso IPPB & $341(87,2)$ \\
Infección necrosante & $39(10)$ \\
Adquisición nosocomial & $9(2,9)$ \\
Patología crónica asociada & $191(48,9)$ \\
Diabetes & $75(19,1)$ \\
Obesidad & $65(16,6)$ \\
Hepatopatía crónica & $51(13,1)$ \\
Insuficiencia cardiaca (IC) & $38(9,7)$ \\
Neoplasia & $32(8,1)$ \\
Enfermedad pulmonar obstructiva & $26(6,6)$ \\
Insuficiencia renal (IR) & $23(5,9)$ \\
Malnutrición & $15(3,8)$ \\
Infección VIH & $12(3,0)$ \\
Factores predisponentes & $299(76,1)$ \\
Puerta de entrada & $207(52,9)$ \\
Insuficiencia venosa & $82(21,2)$ \\
Arteriopatía periférica & $41(10,5)$ \\
Inmunosupresión & $40(10,3)$ \\
Traumatismo & $36(9,2)$ \\
UDVP & $26(6,6)$ \\
Corticoterapia & $21(5,4)$ \\
Institucionalizados & $11(2,8)$ \\
\hline &
\end{tabular}

\section{TABLA II}

PRUEBAS RADIOLÓGICAS REALIZADAS Y RENTABILIDAD DIAGNÓSTICA

\begin{tabular}{lc}
\hline & $N$ (\% estudios diagnósticos) \\
\hline Estudios de imagen & $230(34,3)$ \\
Radiología simple & $24 / 123(19,5)$ \\
Ultrasonografía & $37 / 80(46,2)$ \\
Tomografía computerizada & $12 / 21(57,1)$ \\
Resonancia magnética & $6 / 6(100)$ \\
\hline
\end{tabular}

el 31,7\% ( $\mathrm{n}=124)$. Obtuvimos una tasa de aislamientos del $21 \%$ para hemocultivos $(\mathrm{n}=76)$ y del $73,8 \%$ para cultivos de la lesión $(\mathrm{n}=84)$. Los gram-positivos fueron los microorganismos hallados con mayor frecuencia con un 59,5\%, ninguno de los Staphylococcus aureus aislados fueron resistentes a meticilina. El cultivo fue polimicrobiano en el 1,3\% de los hemocultivos y en $19 \%$ de los cultivos de la lesión. Los

\begin{tabular}{ll}
\multicolumn{2}{c}{ TABLA III } \\
\multicolumn{1}{c}{ AISLAMIENTOS MICROBIOLÓGICOS OBTENIDOS } \\
\hline Variable & N $\%)$ \\
\hline Gram-positivos & $40(54,8)$ \\
Staphylococcus aureus & $21(28,3)$ \\
Streptococcus sp & $19(27,2)$ \\
Streptococcus pyogenes & 5 \\
Enterococcus faecalis & 3 \\
S. constellatus & 2 \\
S. agalactiae & 2 \\
S. oralis & 1 \\
S. viridans & 1 \\
Otros grupos & 5 \\
Gram-negativos & $23(31,5)$ \\
Escherichia coli & 6 \\
Morganella morganii & 3 \\
Pseudomona aeruginosa & 3 \\
Proteus mirabilis & 3 \\
Serratia marcescens & 1 \\
Acinetobacter baumannii & 1 \\
Aeromona hydrophila & 1 \\
Stenotrophomona maltophilia & 1 \\
Citrobacter freundii & 1 \\
Haemophilus influenzae & 1 \\
Klebsiella pneumoniae & 1 \\
Enterobacter cloacae & 1 \\
Anaerobios & 1 \\
Hongos & 1 \\
\hline &
\end{tabular}

TABLA IV

CARACTERÍSTICAS DE LOS EPISODIOS INCLUIDOS EN EL ESTUDIO

\begin{tabular}{ll}
\hline Variable & $N(\%)$ \\
\hline Antibióticos sistémicos & $370(94,6)$ \\
Duración media (días) & 12,3 Mediana 10 \\
Monoterapia & $198(50,6)$ \\
Número medio de fármacos por ingreso & 1,7 \\
Intervenciones quirúrgicas & $169(43,2)$ \\
Drenaje simple & $58(34,3)$ \\
Desbridamiento & $93(55,0)$ \\
Amputación-Reconstrucción & $9(5,3)$ \\
Otros & $9(5,3)$ \\
\hline
\end{tabular}

microorganismos hallados se resumen en la Tabla III. Los pacientes a los que se les practicaron hemocultivos presentaban una temperatura media de ingreso mayor que a los que no se les realizó esta prueba $\left(37,8 \pm 1,1^{\circ} \mathrm{C}\right.$ vs. $36,9 \pm 0,9^{\circ} \mathrm{C}$; $\mathrm{p}=0,00125)$. 
Prácticamente la totalidad de los pacientes $(94,6 \%)$ recibieron antibióticos durante su ingreso. En casi la mitad se empleó terapia combinada (media de fármacos $1,7 \pm 0,7$ ) durante más de una semana (mediana 10 días) (Tabla IV). Amoxicilina con ácido clavulánico fue el antimicrobiano más empleado (39\%), seguido de aminoglucósidos $(31,3 \%)$, clindamicina $(25,6 \%)$, cloxacilina $(21,0 \%)$ y fluorquinolonas $(18 \%)$. El 43,2\% precisó además tratamiento quirúrgico en diferentes modalidades (Tabla IV).

Fallecieron 27 pacientes $(6,9 \%)$ de los cuales en 11 $(40,7 \%)$ se podría atribuir al proceso séptico la causa fundamental del éxitus. La edad media de los fallecidos era superior a la de los vivos $(68,5 \pm 16,7$ vs. $48,8 \pm 20,1 \mathrm{p}<$ $0,001)$. Por diagnósticos la fascitis $(n=7)$ se asoció a una mortalidad del 42,8\%, seguida de los abscesos $(\mathrm{n}=88)$ $(8,6 \%)$ y la celulitis $(n=271)(5,5 \%)$. Todos los fallecidos tenían comorbilidad asociada. No obstante, no todas las enfermedades se asociaron a una mortalidad mayor, lo que también se observó sólo para algunos factores predisponentes (Tabla V). Cuando se restringió el análisis exclusivamente a los pacientes con comorbilidad, las únicos pro- cesos que mantuvieron la significación estadística fueron la IC, hepatopatía crónica y la arteriopatía periférica ( $\mathrm{p}<$ 0,0001). Las infecciones consideradas como necrosantes tuvieron una proporción de mortalidad de $23,7 \%$ frente al 4,9\% de las no necrosantes (OR: 6,04 IC95\%: 2,3-15,9; p < 0,0001). Los únicos parámetros analíticos que se asociaron a mayor mortalidad fueron los concentraciones séricas de proteínas totales $(55 \pm 9 \mathrm{~g} / \mathrm{L} v s .6,3 \pm 0,8 \mathrm{~g} / \mathrm{L} ; \mathrm{p}=0,0231)$ y albuminemia $(22 \pm 7 \mathrm{~g} / \mathrm{L} v s .29 \pm 7 \mathrm{~g} / \mathrm{L} ; \mathrm{p}=0,0125)$. No encontramos diferencias estadísticamente significativas en lo que respecta a niveles de tensión arterial, temperatura, leucocitos y neutrófilos, así como tampoco para la creatinina sérica.

\section{DISCUSIÓN}

Existen pocos trabajos epidemiológicos acerca de IPPB en población general, de hecho la mayoría son revisiones (1014), que versan sobre poblaciones específicas, como infecta-

TABLA V

TASA DE MORTALIDAD OBSERVADA SEGÚN TIPO DE ENFERMEDAD CRÓNICA O PREDISPONENTE

\begin{tabular}{|c|c|c|c|c|}
\hline & Exitus $n=27$ & Vivos $n=364$ & ORIC & Significación \\
\hline Comorbilidad & $25(92,6 \%)$ & $166(45,6 \%)$ & $15,8(3,6-98,2)$ & 0,00001 \\
\hline Insuficiencia cardiaca (IC) & $14(51,8 \%)$ & $24(6,6 \%)$ & $15,7(6,1-40,5)$ & $<0,0001$ \\
\hline Hepatopatía crónica & $12(44,4 \%)$ & $39(10,7 \%)$ & $6,8(2,7-16,9)$ & 0,0001 \\
\hline \multicolumn{5}{|l|}{ Malnutrición } \\
\hline$(n=306)$ & $3(23 \%)$ & $12(5,0 \%)$ & $5,7(1,1-27)$ & 0,0033 \\
\hline Insuficiencia renal (IR) & $5(18,5 \%)$ & $18(4,9 \%)$ & $4,3(1,3-14,0)$ & 0,01 \\
\hline Neoplasia & $6(22,2 \%)$ & $26(7,2 \%)$ & $3,8(1,2-11,2)$ & 0,0018 \\
\hline Diabetes & $11(40,7 \%)$ & $64(17,6 \%)$ & $3,3(1,4-8,0)$ & 0,0072 \\
\hline \multicolumn{5}{|l|}{ Obesidad } \\
\hline$(n=286)$ & $7(36,8 \%)$ & $58(21,7 \%)$ & & N.S. \\
\hline Enfermedad pulmonar obstructiva & $3(11,1 \%)$ & $23(6,3 \%)$ & & N.S. \\
\hline Infección VIH & $1(3,8 \%)$ & $11(3,03 \%)$ & & N.S. \\
\hline \multicolumn{5}{|l|}{ Factor predisponente } \\
\hline Arteriopatía periférica & $11(40 \%)$ & $30(8,8 \%)$ & $8,2(3,1-20,8)$ & $<0,0001$ \\
\hline Institucionalizados & $3(12 \%)$ & $8(1,9 \%)$ & $6,5(1,3-30,8)$ & 0,0001 \\
\hline Insuficiencia venosa & $10(40 \%)$ & $72(19,9 \%)$ & $2,5(1,1-5,9)$ & 0,017 \\
\hline Puerta de entrada & $7(25,9 \%)$ & $200(55 \%)$ & $0,29(0,1-0,7)$ & 0,034 \\
\hline Inmunosupresión & $6(22,2 \%)$ & $34(9,4 \%)$ & & N.S. \\
\hline Traumatismo & $4(14,8 \%)$ & $32(8,8 \%)$ & & N.S. \\
\hline UDVP & $2(7,4 \%)$ & $24(6,6 \%)$ & & N.S. \\
\hline Corticoterapia & $3(11 \%)$ & $18(4,9 \%)$ & & N.S. \\
\hline
\end{tabular}




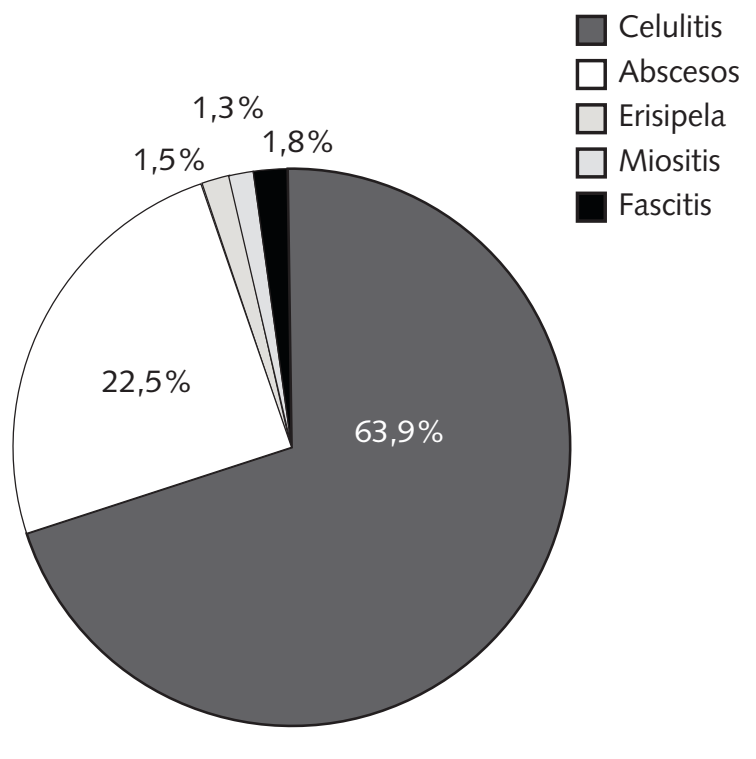

Fig. 1. Distribución por diagnósticos de las diferentes IPPB: Celulitis $63,9 \%$, abscesos $22,5 \%$, erisipela $1,5 \%$, fascitis $1,8 \%$ y miositis $1,3 \%$.

dos por el VIH $(6,15)$ o infecciones necrosantes (16-18). El estudio presentado es el primer trabajo multicéntrico que describe las características de los pacientes que son hospitalizados con IPPB en nuestro país. Así y a tenor de los resultados hallados (Tabla I), vemos que la mayoría de los que son dados de alta con IPPB primaria ingresan por dicho motivo y adquieren la infección en la comunidad. Estos resultados coinciden con los obtenidos en un estudio epidemiológico italiano de IPPB en infectados por el VIH (6). La celulitis constituyen las 2/3 partes del total de hospitalizaciones por IPPB, afectan sobre todo a miembros, y a cabeza y cuello, lo que se debe a la inclusión de pacientes con infecciones odontogénicas (Figs. 1 y 2).

La incidencia de infección necrosante en nuestro trabajo es del 10\%, algo inferior al 14\% hallado en otra serie nacional (2). Para interpretar este hecho tenemos que considerar primero que la serie de Carratalá y cols. sólo incluye celulitis, y segundo que bajo la acepción de infección necrosante de piel y partes blandas se incluyen numerosos procesos y denominaciones. Para solventar este problema de nomenclatura, algunos autores sugieren utilizar este término exclusivamente a aquellos casos de fascitis necrosante y mionecrosis, añadiendo a la hora de su aplicación adjetivos que indiquen su etiología, localización, microbiología y factores predisponentes (19).

De los resultados obtenidos podemos deducir que en el medio hospitalario se realizan un excesivo número de pruebas de imagen en los pacientes que ingresan con IPPB, aunque estos resultados quizás estén magnificados por el alto porcentaje de celulitis odontogénicas $(22,5 \%)$ a las que en su totalidad se le practicaron ortomopantografías. Como es sabido el diagnóstico de celulitis y otras infecciones de partes blandas es clínico y la realización rutinaria de pruebas radiológicas no está indicada, salvo en caso de duda diagnóstica, sospecha de complicación local (osteomielitis,

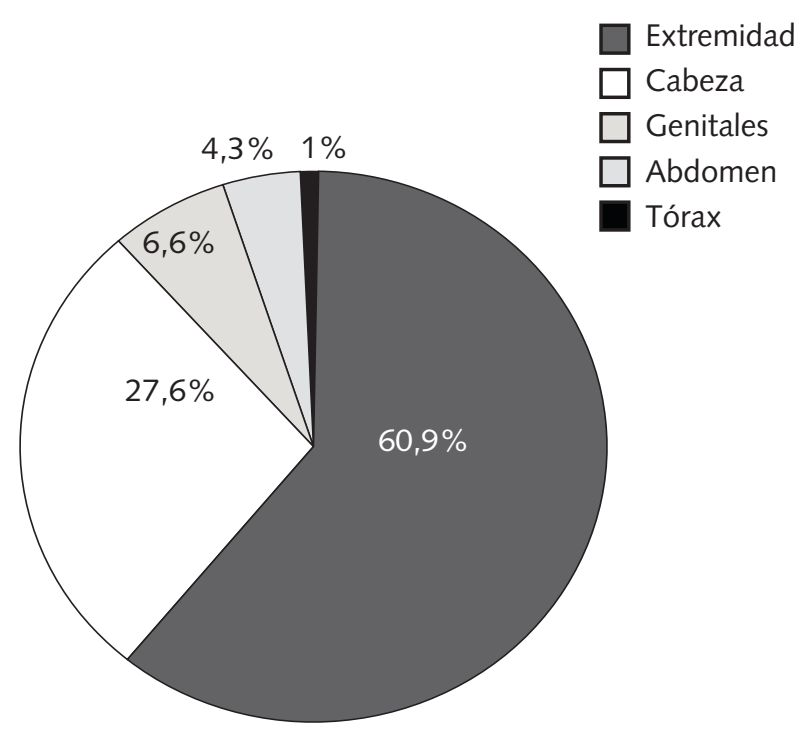

Fig. 2. Localización de las IPPB: Extremidades 60,9\%, Cabeza y cuello 27,6\%, Abdomen 4,3\%, genitales 6,6\% y Tórax 1\%.

absceso, etc.) o infección necrosante (20). Es claro que en un estudio retrospectivo basado en los informes de alta es difícil de valorar si la indicación de la prueba de imagen fue rutinaria o por estos supuestos. Del mismo modo, la baja rentabilidad diagnóstica obtenida en el presente trabajo para las pruebas de imagen, a excepción de RMN que se realizó en un número insignificante de pacientes, refrenda la escasa validez de estas pruebas en el manejo rutinario de las IPPB. Por otra parte la técnica de mayor validez diagnóstica y terapéutica ante la sospecha de infección necrosante de partes blandas es la exploración quirúrgica de la zona, si bien la RMN puede servir para delimitar la profundidad de la lesión (21), no debe demorar nunca el acto quirúrgico si se considera indicado.

El escaso número de infecciones nosocomiales, polimicrobianas o con microorganismos multirresistentes obtenido se debe sin duda, a haber excluido a las infecciones secundarias a cirugía, quemaduras o úlceras de presión. Por otra parte, la proporción de pacientes institucionalizados es pequeña $(2,8 \%)$ lo que sin duda también puede influir en la existencia de una flora con baja tasa de resistencia. Los cocos gram-positivos son los microorganismos encontrados con mayor frecuencia en los aislamientos, como en la mayoría de los estudios publicados $(13,22,23)$. No encontramos mayor morbimortalidad en aquellos pacientes donde se aislaron bacilos gram-negativos en general o Pseudomona en particular como se describe en otra serie (2), ni cuando reducimos el análisis a celulitis e infecciones profundas. Esto puede deberse a que obtenemos un porcentaje inferior de aislamientos.

La toma rutinaria de cultivos de la lesión y sobre todo hemocultivos no es coste-eficaz en celulitis (24) y queda restringida a los casos donde hay linfedema, sospecha de bacteriemia, celulitis orbitaria, bucal, heridas en agua dulce o salada (20). En nuestra serie los pacientes a los que se les 
realizaron hemocultivos estaban febriles, por lo que al contrario de lo comentado para las pruebas de imagen, la indicación de esta prueba en IPPB en nuestro medio parece adecuada.

De los resultados acerca de los aislamientos obtenidos, podemos deducir que las IPPB primarias en nuestro medio son causadas por microorganismos sensibles a antibióticos estándar. No obstante podemos apreciar algunas discrepancias con respecto a lo recomendado en los protocolos de la SEIMC y otros artículos de revisión $(8,20)$. Así se aprecia que en la mitad de los casos empleamos terapia antibiótica combinada $(49,4 \%)$, a pesar que la mayoría de los aislamientos son monobacterianos por gram-positivos. El antibiótico más empleado es la amoxicilina-clavulánico (39\%) en vez de cefalosporinas de primera generación (16,3\%) o cloxacilina $(21 \%)$. Esta hegemonía de la amoxicilina-clavulánico también se describe en otra serie nacional (2). Una razón para explicar estos hechos, podría ser el alto porcentaje de celulitis odontogénicas incluidas en los resultados $(22,5 \%)$, no obstante, si excluíamos a este tipo de infección del análisis se mantenía el uso de amoxicilina clavulánico en el $42 \%$ de los casos. Así y aunque nuestro trabajo puede ser cuestionado en algunos aspectos metodológicos, se refleja la divergencia existente entre la práctica clínica real y las recomendaciones terapéuticas publicadas.

Tras el análisis realizado podemos concluir que las IPPB primarias tienen muy buen pronóstico en sujetos previamente sanos, con buen estado nutricional. Así pues, la mortalidad en hospitalizados con IPPB parece restringirse a pacientes con necrosis cutánea o enfermedades crónicas, con una tasa similar a la publicada por otros autores $(2,16-18)$. Un dato a destacar es que aquéllas patologías que favorecen la malnutrición y los edemas, como son la IC, hepatopatía e insuficiencia renal, conllevan una tasa de mortalidad ostensible (OR:15,7, 6,8, 4,3 respectivamente), inclusive cuando el estudio se limita exclusivamente a los pacientes con enfermedades crónicas. Hecho que también se da en el caso de presentar arteriopatía periférica como lo que se describe para pacientes con pie diabético $(25,26)$.

A modo de conclusión final pensamos que nuestro trabajo describe la realidad de los pacientes ingresados por IPPB en hospitales de nuestro país. Así podemos considerar a las infecciones primarias de piel y partes blandas como un proceso benigno en la mayoría de los casos, y que por tanto los pacientes sanos sin criterios de gravedad pueden ser dados de alta de forma precoz y ser atendidos bajo las nuevas modalidades de hospitalización domiciliaria $(27,28)$. Además este estudio nos ilustra acerca de la variabilidad en la práctica médica con la más que probable sobreutilización de pruebas radiológicas y la discrepancia en la aplicación de los tratamientos recomendados por las guías de práctica clínica, aunque para asegurar esta conclusión sean necesarios estudios prospectivos controlados.

\section{AGRADECIMIENTOS}

A los doctores María José Fernández, Emilia Fernández, José del Río Mata, Antonio Lara, Francias Gálvez Castro, Tomás Padrón Concepción, Jesús María Pena González y demás trabajadores de los Servicios de Documentación Clínica de los centros participantes sin cuya colaboración no habría sido posible la realización de este trabajo.

\section{Bibliografía}

1. Swartz MN. Infecciones de la piel y de los tejidos blandos. En: Mandell GL, Bennet JE, Dollin R, editores. Enfermedades infecciosas. Principios y Práctica. 5a ed. Buenos Aires: Panamericana; 2002, p. 1258-1283.

2. Carratala J, Rosón B, Fernández-Sabé N, Shaw E, del Rio O. Rivera A, et al. Factors associated with complications and mortality in adult patients hospitalized for infectious cellulitis. Eur J Clin Microbiol Infect Dis 2003; 22: 151-157.

3. Wilson SE, Solomkin JS, Le V, Cammarata SK, Bruss JB. A severity score for complicated skin and soft tissue infections derived from phase III studies of linezolid. Am J Surg 2003; 185: 369-75.

4. Kuncir EJ, Tillou A, St Hill CR, Petrone P, Kimbrell B, Asensio JA. Necrotizing soft-tissue infections. Emerg Med Clin North Am 2003; 21: $1075-87$

5. Headley AJ. Necrotizing soft tissue infections: a primary care review. Am Fam Physician 2003; 68: 323-8.

6. Manfredi R, Chiodo F. Cellulitis and soft tissue infection in patients with HIV disease: epidemiological and microbiological features. Infez Med. 2001; 9:101-7.

7. Instituto Nacional de Estadística. Disponible en: http::// www.ine.es.

8. Guerrero A, Ariza J, Gomis M, Barberán J, Sánchez C, Barros C, Infecciones osteoarticulares y de partes blandas. Protocolos Clinicos de la SEIMC. www/seimc.org

9. Memoria del Servicio Andaluz de Salud INIHOS 2002. Consejería de Salud. Junta de Andalucía.
10. Nichols RL, Florman S. Clinical presentations of soft-tissue infections and surgical site infections. Clin Infect Dis 2001; 33: S8493.

11. Stone HH. Soft tissue infections. Am Surg 2000; 66: 162-5.

12. Lewis RT. Soft tissue infections. World J Surg 1998; 22: 146-51.

13. Simmen HP, Giovanoli P, Battaglia H, Wust J Meyer VE. Soft tissue infections of the upper extremities with special consideration of abscesses in parenteral drug abusers. A prospective study. J Hand Surg 1995; 6: 797-800

14. McHenry, C R. Compton, C N. Soft Tissue Infections. Problems gen Surg 2002; 19:7-19.

15. Manfredi R, Calza L, Chiodo F. Epidemiology and microbiology of cellulitis and bacterial soft tissue infection during HIV disease: a 10 year survey J Cutan Pathol 2002; 29: 168-72.

16. Singh G, Sinha SK, Adhikary S, Babu KS, Ray P, Khanna SK. Necrotising infections of soft tissues a clinical profile. Eur J Surg 2002; 6: 366-371.

17. Hsiao GH, Chang CH, Hsiao CW, Fanchiang JH, Jee SH. Necrotising soft tissues infections. Surgical or conservative treatment? Dermatol Surg 1998; 2: 243-7.

18. Brandt MM, Corpron CA, Wahl WL. Necrotizing soft tissue infections: a surgical disease. Am Surg 2000; 10: 967-70.

19. Sánchez U, Peralta G. Infecciones necrosantes de partes blandas: nomenclatura y clasificación. Enferm Infecc Microbiol Clin 2003; 21: 196-199. 
20. Swartz MN. Cellulitis. N Eng J Med 2004; 350: 904-12.

21. Schmid MR, Kossmann T, Duewell S. Differentiation of necrotizing fasciitis and cellulites using MR imaging. Am J Roentgenol 1998; 170: $615-20$

22. Sader HS, Jones RN, Silva JB; SENTRY Participants Group (Latin America). Skin and soft tissue infections in Latin American medical centers: four-year assessment of the pathogen frequency and antimicrobial susceptibility patterns.Diagn Microbiol Infect Dis 2002; 44: 281-8.

23. Rennie RP, Jones RN, Mutnick AH: SENTRY Progran Study Group (North America). Ocurrence and antimicrobial susceptibility patterns isolated from Skin and soft tissue infections: report from SENTRY Antimicrobial Surveillance Program (United States and Canada 2000) Diagn Microbiol Infect Dis 2003; 4: 287-93.

24. Perl B, Gottehrer NP, Ravek D, Sclesinger Y, Rudensky B, Yinnon
AM. Cost-effectiveness of blood cultures for adult patients with cellulites. Clin Infect Dis 1999; 29: 1483-8.

25. Benotmane A, Mohammedi F, Ayad F, Kadi K, Azzouz A. Diabetic foot lesions: etiologic and prognostic factors. Diabetes Metab 2000; 2: 113-7.

26. Lavery LA, van Houtum WH, Harkless LB. In-hospital mortality and disposition of diabetic amputees in The Netherlands. Diabet Med 1996; 13: 192-7.

27. Dalovisio JR, Juneau J, Baumgarten K, Kateiva J. Financial impact of home intravenous antibiotic program on Medicare managed care program. Clin Infect Dis 2000; 30: 639-42.

28. Willians DN, Rehm SJ, Tice AD, Bradley JS, Kind AC, Craig WD. Practice guidelines for community-based parenteral anti-inffective therapy. ISDA Practice Guidelines Committee. Clin Infect Dis 1997; 25: 787-801. 